\title{
Origine(s) berbère(s) : Linguistique et préhistoire
}

Salem Chaker

\section{(2) OpenEdition}

Journals

Édition électronique

URL : https://journals.openedition.org/encyclopedieberbere/2829

DOI : 10.4000/encyclopedieberbere.2829

ISSN : 2262-7197

\section{Éditeur}

Peeters Publishers

\section{Édition imprimée}

Date de publication : 1 juin 2013

Pagination : 5819-5844

ISBN : 978-2-7584-0184-1

ISSN : 1015-7344

\section{Référence électronique}

Salem Chaker, «Origine(s) berbère(s) : Linguistique et préhistoire », Encyclopédie berbère [En ligne], 35 | 2013, document O26, mis en ligne le 12 mars 2021, consulté le 17 février 2022. URL : http:// journals.openedition.org/encyclopedieberbere/2829; DOI : https://doi.org/10.4000/ encyclopedieberbere.2829

Ce document a été généré automatiquement le 17 février 2022.

(c) Tous droits réservés 


\title{
Origine(s) berbère(s) : Linguistique et préhistoire
}

\author{
Salem Chaker
}

1 [Cette notice a bénéficié des relectures et commentaires attentifs de deux collaborateurs préhistoriens de l'EB, Colette Roubet (Paris) et Jorge Onrubia-Pintado (Madrid) : qu'ils en soient ici remerciés. J'assume bien entendu seul la responsabilité de ce texte.]

«Et si les Berbères [et leur langue] ne venaient de nulle part?»(Gabriel Camps 1981, p. 20).

\section{Introduction}

2 La question des origines berbères - du peuple et de la langue - a fait couler beaucoup d'encre et, comme le soulignait avec humour Gabriel Camps (1981), depuis les sources antiques, il y a peu de régions d'où on ne les a pas fait venir ! Le linguiste ajoutera qu'il y a peu de langues avec lesquelles on n'a pas cherché à établir une parenté : du celtique au basque en passant par les langues amérindiennes et, bien sûr, le sémitique! Si l'on met de côté les mythes et légendes antiques et médiévaux, très précisément étudiés et mis en perspective par Yves Moderan (cf. sa remarquable notice M152a «Mythes d'origine des Berbères ", EB XXXI, 2010), qui, comme on l'a aussi montré (notice M152b), ont la vie dure, et que l'on se limite aux travaux dits scientifiques depuis le $\mathrm{XIX}^{\mathrm{e}}$ siècle, la palette des thèses est large et les débats ont été et restent vifs.

La thèse classique d'une origine ptoche/moyen-orientale a longtemps prévalu, cette région étant considérée comme le berceau du monde méditerranéen : au plan culturel, de la néolithisation; au plan anthropologique, du peuplement ; et au plan linguistique, $\mathrm{du}$ (chamito)-sémitique (ou afroasiatique). Elle est largement dominante chez les sémitisants, souvent réticents à envisager que le berceau primitif de la famille linguistique chamito-sémitique ait pu se situer ailleurs qu'au Proche/Moyen-Orient. Par voie de conséquence, les langues non-sémitiques de la famille procèderaient nécessairement de la diffusion vers l'Afrique, à date ancienne, du (proto-sémitique à 
partir d'un centre moyen-oriental. On reviendra plus loin sur cette thèse, qui a différentes facettes.

$4 \mathrm{Au}$ cours des 50 dernières années, cette théorie a été puissamment relayée par les préhistoriens de l'Afrique du Nord, à travers la thèse dite "capsienne": les ProtoMéditerranéens ( $=$ "Capsiens"*, du site éponyme Capsa $=$ Gafsa en Tunisie) seraient apparus en Afrique du Nord au VIII ${ }^{\mathrm{e}}$ millénaire avant J.-C., apportant du Moyen-Orient :

- un nouveau type physique (les Proto-méditerranéens remplacent peu à peu le type "Mechta el-Arbi/Afalou"*),

- une nouvelle culture (le Néolithique),

- et une langue (le proto-berbère).

5 La convergence des indices semblait forte et nous y avons nous-même un temps adhéré (Chaker 1984 et notice A247 "Apparentement de la langue berbère »). Pourtant, à y regarder de plus près, la thèse était, dès l'origine, très fragile (cf. infra, $\$$ «L peuplement préhistorique de l'Afrique du Nord »).

6 Plus récemment, chez les linguistes, les tenants d'une origine africaine, dans le cadre de la théorie d'un berceau africain de l'afro-asiatique et de la néolithisation - qui serait le moteur de l'expansion d'un peuple et d'une langue -, ont consolidé leurs positions. Greenberg, Diakonoff, Behrens, Ehret, parmi bien d'autres, ont proposé des localisations et des dynamiques d'expansion à partir de localisations africaines, situées dans le Sahara central ou, plus souvent, en Afrique orientale (Soudan, Nubie-Kordofan, Darfour, Ethiopie...). Ils reprennent d'ailleurs, sur des bases renouvelées, une idée fort ancienne qui remonte à la fin XIX ${ }^{\mathrm{e}}$ siècle, celle d'une liaison entre certains groupes de langues africaines et langues "chamitiques ", déjà avancée par des africanistes comme Carl Meinhoff (1857-1944).

7 Il n'est évidemment pas fortuit que les sémitisants adoptent généralement la thèse de l'origine proche-ou moyen-orientale des langues chamito-sémitiques, alors que les "afro-asiatisants » optent pour une localisation africaine du berceau primitif. La position des premiers est manifestement surdéterminée par le poids historique et culturel des civilisations sémitiques anciennes et la diffusion large des langues qui en ont été les vecteurs (akkadien, phénicien, "sud-arabique", arabe...) ; ils projettent ainsi à rebours, sur des temps préhistoriques totalement obscurs, les dynamiques culturelles et linguistiques connues des temps protohistoriques ou historiques (expansion phénicienne, sémitique et arabe, influence des civilisations mésopotamiennes et diffusions des religions monothéistes). Or, cette extrapolation/rétroprojection est fragile, et même sans fondement positif: au cours des temps préhistoriques (paléolithique moyen, supérieur et néolithique), aux plans culturel et anthropologiques, l'Afrique du Nord, a été nettement plus liée à l'Europe du Sud ${ }^{1}$ (Péninsule ibérique, Italie...) qu'avec le Proche et Moyen-Orient.

8 Les seconds sont sans doute influencés par «l'air du temps" qui voit dans l'Afrique, notamment l'Afrique de l'Est, le berceau de l'humanité. L'argument principal à l'appui de cette thèse est d'ailleurs inscrit dans la nouvelle dénomination "afro-asiatique » introduite par J. Greenberg $(1955,1963)$ : sur les cinq branches (six pour certains, qui y rajoutent une branche africaine omotique) de la famille afro-asiatique : quatre sont africaines (berbère, égyptien, couchitique, tchadique), une seule est «asiatique », le sémitique, que l'on serait donc en droit de considérer comme une "excroissance géographique ", une projection à partir d'un berceau originel africain. Sympathique volonté de réhabilitation du continent « oublié », mais là-aussi risque d'anachronisme - 
l'émergence et la diffusion d'Homo (sapiens) n'a rien à voir avec des mouvements de populations beaucoup plus récents - et faiblesse des arguments et des données concrètes étayant cette thèse. "L'évidence géographique " notamment paraît assez inconsistante : que ne pourrait-on tirer comme conclusions bizarres de la répartition de la langue anglaise dans le monde actuel si l'on n'en connaissait l'histoire: l'Angleterre serait certainement considérée comme une projection lointaine d'un centre originel situé entre Pacifique et Atlantique, sans doute en Amérique du Nord, dans un arc d'expansion allant de l'Afrique du Sud, à l'Angleterre en passant par l'Australie et l'Amérique du Nord !

Des arguments linguistiques, archéologiques et ethnologiques divers ont été mobilisés pour défendre ces différentes thèses. Mais les données linguistiques berbères n'ont, pendant longtemps, que très rarement été prises en considération, ou que de façon très marginale. Certes, dans les travaux les plus récents, il y a recours plus systématique aux matériaux linguistiques berbères, notamment lexicaux ; mais les comparaisons, mises en relation ou utilisations dans le cadre de démonstrations historiques sont souvent problématiques, voire inacceptables : les formes mobilisées sont rarement soumises à analyse diachronique interne au berbère, ce qui conduit à utiliser des données nonvalides ou inappropriées. Et à fonder les démonstrations sur du sable... Cette faiblesse est particulièrement sévère dans toutes les approches "extensives " qui utilisent un matériel lexical brut. C'est ainsi que la majorité des rapprochements berbère/tchadique de Greenberg (1966) ne résiste absolument pas à la critique du berbérisant : un examen attentif (Bynon 1985) des matériaux lexicaux utilisés par Greenberg conduit à écarter plus de la moitié des rapprochements proposés : 15 seulement sur les 32 avancés sont retenus comme valables - et selon nous, on peut être beaucoup plus sévère et en retenir moins d'une dizaine. On verra qu'il y en va de même pour d'autres théories extensives comme celle de H. G. Mukarovsky à propos du basque et du berbère.

10 Même dans les travaux les plus récents (Blench 2001, Louali \& Philippson 2004...) - qui s'appuient sur une documentation lexicographique bien plus à jour et plus solide -, il n'y a pas d'étude critique interne préalable du matériel mis en œuvre. Toutes ces approches soulèvent le même problème méthodologique : on développe des thèses de linguistique historique impliquant des matériaux berbères sans que la reconstruction interne, morphologique et sémantique, ait été faite.

11 Cette notice propose une synthèse critique des thèses relatives à la mise en place du peuplement et de la langue berbères. Après un rappel des données préhistoriques actuellement acquises ou considérées comme probables, on interrogera le matériel linguistique pour y rechercher des indices allant dans un sens (allochtonie) ou dans l'autre (autochtonie). Mais auparavant, quelques clarifications, rappels conceptuels et méthodologiques s'imposent.

\section{Quelques rappels et mises au point préalables}

12 Pourquoi la question des origines des Berbères est-elle posée, dans cette formulation globalisante, avec une telle constance ? L'inanité de telles problématiques est patente : l'immensité de leur territoire et le caractère particulièrement tourmenté de leur histoire - sans même parler de leur préhistoire -, faite depuis au moins trois millénaires de conquêtes, invasions et apports extérieurs les plus divers - orientaux, 
européens, africains - rend d'emblée un tel questionnement assez inconsistant, s'il n'est pas très précisément explicité et délimité.

Alors que depuis longtemps déjà, l'anthropologie physique a établi qu'il n'existe pas un type humain "berbère", pas même un type dominant (cf. notice A234 "Anthropologie»), mais des variétés régionales et individuelles nombreuses, la question de "l'origine des Berbères" continue néanmoins à être posée, sous cette forme, de manière récurrente, dans l'opinion publique comme dans le champ scientifique. Gabriel Camps (1974, p. 162) insistait déjà sur la diversité du peuplement du Maghreb au VIII ${ }^{e}$ millénaire avant J.-C. Ici même, la contribution de Jorge OnrubiaPintado (notice 027) rappelle opportunément que « le tableau susceptible d'être brossé à partir des restes humains néolithiques qui ont fait l'objet d'une étude plus ou moins approfondie est celui d'une population bigarrée ».

On verra bien sûr dans cette interrogation lancinante sur "l'origine des Berbères » l'écho, direct ou indirect, conscient ou inconscient, de la permanence d'enjeux idéologiques, voire directement politiques, considérables liés à cette question (cf. notre notice Ml52b).

15 Par ailleurs, des approximations tenaces interfèrent très souvent dans l'approche de cette question des origines. D'abord parce que les a prioris sont omniprésents, y compris dans le champ scientifique; aussi parce que les raccourcis dans les argumentations abondent, cette question étant à la croisée de disciplines diverses : préhistoire, anthropologie physique/génétique, anthropologie culturelle, linguistique... Le spécialiste de chacune de ces disciplines sera souvent tenté de s'appuyer sur des données empruntées aux disciplines voisines, sans en avoir une maîtrise globale, en ayant tendance à les durcir, à prendre de simples hypothèses pour des résultats acquis, alors que bien souvent, elles sont déjà obsolètes, ou purement conjecturales ou non admises par la majorité des spécialistes du champ scientifique concerné... On ne perdra pas de vue que dans toutes les disciplines impliquées, la diversité des thèses et hypothèses est grande, et la marge d'incertitude considérable; même sur des questions cruciales d'anthropologie préhistorique le débat est permanent et les thèses « admises » sont renouvelées quasiment toutes les décennies ${ }^{2}$.

On soulignera aussi que technicité et/ou érudition n'est pas du tout un gage de validité scientifique. La plupart des disciplines impliquées mettent en œuvre des méthodes et techniques d'investigation hautement sophistiquées - la génétique de populations, les techniques de datation absolue, la glottochronologie... Mais la technicité ne constitue pas en elle-même un élément de démonstration probant : ce n'est qu'un outil dans une chaîne argumentative et les conclusions restent totalement dépendantes des hypothèses de départ, des conditions de mise en œuvre et de l'interprétation des résultats, aspects qui eux sont toujours fortement déterminés par «les opinions ambiantes ", l'idéologie, les opinions individuelles, voire les positions et enjeux académiques... La réflexion critique, a priori et a postériori, est indispensable et il serait tout à fait illusoire de penser que la question des origines pourrait trouver une réponse univoque et satisfaisante par la simple mobilisation de moyens techniques, fussent-ils ceux de la génétique moléculaire ${ }^{3}$; comme nous le signale C. Roubet (in litt.), «les critiques internes portant sur le matériel génétique animal restent fortes, et que dire alors du matériel génétique humain, quantitativement moins nombreux, et qui n'est soumis qu'avec parcimonie aux investigations génétiques en raison d'une grande prudence politico-scientifique dans la plupart des pays ». Le linguiste rappellera à ce 
propos la grande fragilité et les limites absolues, mises en évidence depuis fort longtemps (cf. Penchoen 1968), de la glottochronologie dont l'utilisation dans le domaine afro-asiatique est des plus problématiques, à la fois parce que l'échelle des temps potentiellement impliqués dépasse largement les limites mathématiques de la méthode mais aussi du fait des contacts et échanges anciens et incessants entre langues de la famille.

Cette question des «origines » et «filiations » des groupes humains exige donc une grande prudence et on renverra une fois encore à Gabriel Camps qui, qu'elles aient été ses convictions et positions sur le sujet, invitait à la pondération sur le sujet :

"Quand on fait le bilan de nos misérables connaissances des industries préhistoriques, on comprend combien ces recherches [sur les origines] sont spéculatives et souvent gratuites... » (1974, p. 190).

Il n'est pas non plus superflu de rappeler qu'il n'y a aucun lien nécessaire entre génétique, langue et culture. La chose est tellement évidente - il suffit d'ouvrir les yeux et les oreilles sur le monde contemporain - qu'on ne reviendrait pas sur une telle proposition, qui peut apparaitre comme un truisme, si on ne rencontrait très fréquemment, sous la plume d'éminents spécialistes, des thèses et formulations qui laissent penser ou affirment carrément le contraire.

- On peut parler la même langue ou des langues apparentées sans du tout avoir le même patrimoine génétique. Comme tout fait de culture, les langues voyagent, s'empruntent et peuvent être imposées à de nouvelles populations et devenir l'attribut de groupes humains génétiquement très différents. Et l'interférence génétique entre les groupes concernés peut tout à fait être marginale, infime même : la diffusion des langues et les phénomènes de substitution linguistique, même à grande échelle, n'impliquent pas nécessairement la submersion démographique d'un groupe par un autre. Chacun sait, par exemple, que la grande majorité des arabophones de l'Afrique du Nord actuelle sont d'anciens Berbères ayant adopté la langue arabe - en tout cas pour ceux des zones rurales, qui représentaient encore il y a 50 ans plus de $85 \%$ de la population. La diffusion et la substitution linguistiques reposent fondamentalement sur la domination, réelle et/ou symbolique, non sur les équilibres démographiques : concrètement, ce ne sont pas toujours les plus nombreux qui imposent leur langue.

- Symétriquement, des populations peuvent être génétiquement très proches, voire strictement identiques, et parler des langues totalement différentes, n'appartenant même pas à la même famille linguistique : parmi les Scandinaves, les Suédois et les Norvégiens parlent des langues germaniques, indo-européennes, alors que les Finlandais parlent une langue finno-ougrienne qui n'a pas de lien avec celles de leurs voisins ; même situation pour les Baltes : les Estoniens parlent une langue finno-ougrienne, les Lithuaniens et les Lettons des langues indo-européennes... On sera pourtant bien en peine de distinguer au plan de l'apparence physique un Finlandais d'un Suédois ou un Estonien d'un Lithuanien.

- Il en va de même pour la culture en général, matérielle ou immatérielle : identité ou parenté de langue n'implique aucunement proximité des cultures et, inversement, communauté de culture n'implique pas communauté linguistique et/ou génétique. Le monde iranien fait partie, au plan de la langue, du domaine indoeuropéen; culturellement, il est une composante de l'aire arabo-islamique et n'a pas (plus) grand-chose à voir avec la Scandinavie germanique, au point que l'opinion publique, même éclairée, identifie souvent les Iraniens comme des «Arabes »... La même chose peut être dite du sous-continent indien où prédominent les langues indo-européennes mais dont la culture n'a pas (ou plus) grand- 
chose à voir avec celle des «Indo-européens" d'Europe. Qui, eux-mêmes, ont, pour l'essentiel, adopté une culture religieuse d'origine sémitique, “judéo-chrétienne”... objets, les décors voyagent et s'empruntent continuellement et ceci, sans le moindre doute, depuis l'aube de l'humanité. Il n'y a aucune raison qu'un groupe humain ne s'approprie pas, à l'occasion de contacts même sporadiques, un objet, une technique, voire un rite, qui lui apporte un gain d'efficacité, une satisfaction, matérielle ou morale, nouvelle. La diffusion des objets culturels n'implique pas nécessairement diffusion linguistique et/ou génétique.

villera donc à ne pas faire dire à la notion de «culture » ou " civilisation » au sens où les préhistoriens emploient ces termes plus qu'elle ne signifie. Le concept de " culture ibéromaurusienne » ou " capsienne » ou tout autre ne nous apprend strictement rien sur la langue des populations concernées. Ces "cultures préhistoriques", qui sont identifiées exclusivement par des objets, des techniques, des pratiques laissant une trace matérielle (alimentation, rites funéraires, décors...), peuvent parfaitement se diffuser sur des aires gigantesques et être adoptées par des populations de langues et de patrimoines génétiques totalement différents.

Il est à peu près impossible de tirer quelque conclusion tranchée que ce soit quant à la langue à partir des données culturelles ou anthropologiques/génétiques. Tout au plus peut-on les considérer comme des indices ouvrant la porte à des hypothèses.

La conséquence de ces rappels et mises au point s'impose d'elle-même : le fait que la langue berbère appartienne indiscutablement à la famille chamito-sémitique/afroasiatique :

- N'implique pas que les Berbères soient génétiquement apparentés aux/à d'autre populations de langues afro-asiatiques ou en soient issus, ni que celles-ci, prises dans leur globalité, soient homogènes au plan génétique ;

- Qu'ils aient nécessairement migré vers l'Afrique du Nord à partir d'une autre région du «monde afro-asiatique».

C'est une possibilité, mais cela ne peut être posé comme un postulat ou une donnée acquise, comme semblent le penser la plupart des auteurs, anciens et récents, tenants de la thèse "moyen-orientale » ou de la thèse " est-africaine ». C'est tout au plus une hypothèse de travail.

\section{Le peuplement de l'Afrique du Nord : les données de la préhistoire}

Sans nous aventurer trop avant sur les terrains complexes et encore très débattus des peuplements paléolithiques, anciens et moyens, de l'Afrique du Nord, les travaux les plus récents convergent vers quelques constats et hypothèses fortes ${ }^{4}$ :

- La très grande ancienneté de la présence humaine en Afrique du Nord (1,8 millions d'années) ;

- La permanence de cette présence humaine depuis ces périodes reculées (il n'y a pas de " période vide »);

- Une très probable continuité anthropologique au cours des temps paléolithiques : «Les données disponibles sur les fossiles humains plaident en faveur d'une évolution sur place des populations d'Homo sapiens en Afrique du nord-ouest. Du 
point de vue paléoanthropologique, il n'y aurait donc pas de rupture entre les populations anciennes (Djebel Irhoud) et les populations plus récentes (Taforalt), les fossiles atériens découverts dans la région de Témara représentant une phase intermédiaire au sein de cette évolution..." (Préhistoire du Maghreb 2011, t. I : Nespoulet et al., p. 146);

continuité pouvant même aller localement jusqu'au peuplement contemporain (Préhistoire du Maghreb, 2011, t. I, notamment : Coppa et al. ; Kefi-Ben Atig \& BéraudColomb).

\section{Sur le peuplement autochtone récent de l'Afrique du Nord}

Pour ce qui est des temps épipaléolithiques et néolithiques, on dispose de données globales assez solides sur le peuplement de l'Afrique du Nord; on peut même avancer quelques éléments de certitude : les populations d'Homo sapiens sapiens constituées de Cro-Magnons africains du type "Mechta-Afalou" (ou "Mechta el-Arbi"*) porteurs de la culture "ibéromaurusienne" sont en place à partir de - 20000 BP et couvrent de manière homogène l'ensemble de l'Afrique du Nord tellienne. À partir de $-8000 \mathrm{BC}$ apparaissent, d'abord sur un territoire plus continental et plus méridional, des Protoméditerranéens, associés à la culture dite "capsienne", qui remplacent progressivement les premiers et qui semblent être à l'origine de l'essentiel du peuplement " méditerranéen » actuel.

Il n'y a pas de rupture chronologique ou anthropologique, ni de disjonction "sociologique » radicales entre les "Ibéromaurusiens" et les "Capsiens" 5 . Il est établi que les Ibéromaurusiens et les Capsiens ont vécu de manière concomitante et parfois sur les mêmes sites; les auteurs qui se sont penchés récemment sur la configuration globale du peuplement et des cultures en Afrique du Nord à la fin des temps paléolithiques et au début du Néolithique mettent en évidence la coexistence et le métissage, anthropologique et culturel, plutôt que le conflit et l'isolement: «D'autre part, les données disponibles sur l'Holocène n'enregistrent ni conflits, ni faits d'isolement très strict... » (Roubet et al., 2011). Ce qui jette d'emblée le doute sur la thèse de deux populations nettement distinctes et donc sur celle d'une "importation du proto-berbère » par des immigrants de culture capsienne. Par ailleurs, l'espace primitif capsien est assez réduit par rapport à celui de l'ibéromaurusien et, assez bien délimité : alors que le premier est clairement tellien, de l'Est algérien au Maroc, le second est « essentiellement continental. C'est une civilisation des Hautes-Plaines. " (Grébénart, notice C 20, EB XII, p. 1760).

On rappellera que Gabriel Camps, dans son ouvrage de synthèse de 1974 (p. 190-194), tout en souscrivant à la thèse d'une origine extérieure de ces Proto-méditerranéens capsiens, le fait avec une grande prudence et après avoir précisément exposé les deux autres thèses existantes: celle d'une origine européenne (Italie/Sicile : Vaufrey), celle d'une origine est-africaine (Balout, notamment). Et il conclut sa revue critique des trois hypothèses, dont aucune ne lui apparait radicalement indéfendable, de manière très mesurée :

« Nous trouvons donc dans le capsien, dont l'origine première semble bien être le

Proche-Orient, des affinités méditerranéennes, africaines et orientales... ».

En réalité, il n'existe aucune donnée décisive qui permette de faire venir de l'extérieur ces Proto-Méditerranéens d'Afrique du Nord. Il n'y a pas de continuum anthropologique ou culturel avec les Proto-méditerranéens du Moyen-Orient (Natoufiens) ou avec l'Afrique de l'Est. Même si G. Camps semblait l'exclure absolument («Il semble 
impossible que le type humain méditerranéen se soit différencié à partir des hommes de Mechta el-Arbi ", 1974, p. 190), au regard de la faiblesse des arguments en faveur d'une arrivée de l'extérieur, la thèse d'une évolution anthropologique in situ paraît au moins aussi plausible que celle d'une migration. La régression du type anthropologique "Mechta el-Arbi/Afalou" a été très lente et a laissé des traces encore décelables dans le peuplement actuel ${ }^{6}$, donnant ainsi plus l'impression d'une mutation progressive que d'une submersion d'une population par une autre. En tout état de cause, la question de l'origine des Proto-méditerranéens nord-africains ne peut être dissociée de celle, plus globale, du peuplement de l'ensemble de la périphérie méditerranéenne, Nord, Sud et Est, puisque «ce type humain se retrouve sur les trois continents qui bordent la Méditerranée » (Camps, ibid.). Cet aspect du problème, et sa complexité, notamment ses éventuelles implications linguistiques, ne semblent pas avoir été pleinement mesurés.

On peut certes envisager que de petits groupes de Proto-méditerranéens capsiens, porteurs du proto-berbère, se soient mélangés aux Ibéromaurusiens et leur aient imposé leur langue. Mais la thèse inverse est tout aussi défendable : les Capsiens ont été absorbés par les Ibéromaurusiens "autochtones" dont ils ont adopté la langue. On verra plus loin que les données linguistiques ne militent pas en faveur d'une diffusion relativement récente (néolithique) du berbère à l'ensemble de l'Afrique du Nord. Mais surtout, il conviendrait d'établir l'origine extérieure des Capsiens sur des bases archéoanthropologiques qui autoriseraient une filiation avec des Proto-méditerranéens plus anciens, Natoufiens ou autres.

En fait, il s'agit d'un postulat qui repose sur le raccourci d'argumentation suivant : il existe des Proto-méditerranéens en cours de néolithisation (Natoufiens) au Moyen Orient, autour $d u X^{e}$ millénaire; des Proto-méditerranéens sont identifiés en Afrique du Nord à partir du VIII millénaire : les seconds doivent donc dériver des premiers.

Or, au moins deux indices culturels ${ }^{7}$ cruciaux - la néolithisation et l'art - tendent à montrer qu'une telle filiation est quasiment impossible.

\section{Sur la culture néolithique}

32 Les travaux récents tendent à faire remonter très haut le début de la néolithisation en Afrique du Nord et au Sahara, la repoussant à des dates qui la rendent quasiment contemporaine des néolithiques les plus anciens du Moyen-Orient ou d'Afrique de l'Est, du moins si l'on retient la céramique comme paramètre de la néolithisation. Ils tendent aussi à montrer que la néolithisation fut un phénomène complexe, différencié, régionalisé et graduel, et non une "vague unique ", qui aurait submergé l'Afrique du Nord... Les recherches les plus récentes montrent aussi qu'il a existé des pôles nordafricains autonomes très anciens de domestication de certaines espèces animales (bovins) et de plantes (mil, millet, sorgho) : on se reportera au très important dossier «Néolithisation - Néolithique " réuni dans le fascicule XXXIV de l'EB (notices N40 à N49).

Surtout, il est impossible d'attribuer aux Proto-méditerranéens capsiens l'importation de la néolithisation car les formes les plus anciennes de la culture capsienne appartiennent encore clairement au paléolithique :

«Les Capsiens vivaient en prédateurs; il n'existe aucune preuve d'une agriculture rudimentaire ou de l'élevage de certaine espaces» (Grébénart, C20, EB XII, p. 1765-1766)... 
La néolithisation des Proto-méditerranéens s'est donc faite in situ et ne résulte pas de l'importation d'une culture néolithique déjà constituée (cf. Grébénart, ibid., p. 1767 et le dossier "Néolithisation - Néolithique », EB XXXIV). Par voie de conséquence, la thèse d'une importation du proto-berbère en Afrique du Nord par des Proto-méditerranéens grâce à la supériorité que leur aurait donné la maîtrise de techniques néolithiques n'a aucun fondement. Cela n'exclut d'ailleurs pas qu'ils aient néanmoins été les porteurs/ diffuseurs du proto-berbère, mais le processus de néolithisation et de diffusion linguistique serait alors essentiellement local et non importé.

\section{Sur l'art préhistorique}

dernières découvertes (Hachi 2000, 2002, 2003) ${ }^{8}$ remettent totalement en cause la thèse selon laquelle les Proto-Méditerranéens capsiens seraient les inventeurs (et implicitement les importateurs) de l'art en Afrique du Nord; des figurines de terre, datées de - 15 à - 17.000 (BP), ancrent clairement l'art nord-africain dans l'Ibéromaurusien et anéantissent la thèse d'un lien entre «la naissance de l'art et la civilisation capsienne ", encore défendue par Camps (1974, p. 172 et sq.), et a fortiori entre apparition de l'art et un néolithique exogène.

Des critiques et réserves non moins sévères peuvent être formulées à propos des "thèses africaines", qui ne sont étayées par aucun faisceau d'indices solides, anthropologiques et culturels. Au contraire, au cours du paléolithique, moyen et supérieur, et du néolithique, les mouvements culturels et anthropologiques lourds décelables, sont tous dans le sens Nord > Sud, et manifestent donc plutôt des dynamiques d'expansion à partir de l'Afrique du Nord vers le Sahara et l'Afrique sahélienne : c'est notamment le cas de la civilisation atérienne ${ }^{9}$ (cf. Tillet : 1997 et « à paraître ») au paléolithique moyen et, au néolithique, de la descente progressive des « Méditerranéens » vers le Sahara (à partir du IV ${ }^{e}$ millénaire). Par ailleurs, la dimension strictement anthropologique du problème semble souvent quelque peu occultée ou «oubliée ${ }^{10}$ »: on voit mal comment l'essentiel du peuplement, méditerranéen leucoderme, de l'Afrique du Nord pourrait, même sur 10 à 20000 ans, avoir une origine sub-saharienne, donc de type négro-africaine mélanoderme... Difficulté anthropologique que confirment d'ailleurs les analyses génétiques récentes qui établissent qu'il n'y a pas, à-13000 BP, d'affinité entre population nord-africaine et Afrique sub-saharienne et donc que les apports génétiques africains, actuellement décelables, sont postérieurs à cette date :

«L'étude du polymorphisme de l'ADN mitochondrial des 23 spécimens [...] montre que le patrimoine génétique de la population de Taforalt est constitué de deux composantes : une composante eurasiatique [...] et une composante nord-africaine. Aucun polymorphisme subsaharien n'a été observé chez les spécimens de la population de Taforalt. De même, aucune séquence de type subsaharien n'a été retrouvé chez 9 spécimens prélevés du site ibéromaurusien d'Afalou. Ainsi l'hypothèse d'une origine subsaharienne des Ibéromaurusiens est rejetée. Nos résultats montrent plutôt un peuplement de type méditerranéen dans le Nord de l'Afrique au moins jusqu'à 13.000 ans. » (Kefi-Ben Atig \& Béraud-Colomb, in Préhistoire maghrébine, t. I, 2011, p. 238).

Sur la base des données actuellement établies, on peut donc affirmer que les thèses qui font venir les Berbères/le berbère d'un "ailleurs", africain ou moyen-oriental, restent de pures hypothèses; on ne peut évidemment les exclure en tant que telles, mais 
aucune donnée concrète solide ne vient pour l'instant les étayer. Quand bien même l'on parvenait un jour à démontrer l'origine extérieure des Proto-méditerranéens :

- Leur diffusion à l'ensemble de l'Afrique du Nord a été très progressive (cf. supra et notice C20 et Camps 1974) - et s'est opérée sur un substrat anthropologique ibéromaurusien antérieur bien installé, avec lequel ils se sont nécessairement mélangés. Leur apport génétique a donc été en tout état de cause partiel.

- Leur identification comme population porteuse du proto-berbère n'en resterait pas moins une simple hypothèse, dépendant totalement de la théorie que l'on retient quant au berceau originel de l'afro-asiatique. Et, on l'a rappelé, les thèses sont diverses et contradictoires à ce sujet.

\section{Les données linguistiques}

Existe-t-il des traces positives d'un substrat pré-berbère en Afrique du Nord? Traces qui pourraient étayer la thèse d'une origine extérieure de la langue. A ce jour, aucune donnée sociolinguistique (témoignages anciens), linguistique proprement dite ou onomastique (notamment toponymique), n'a jamais permis d'établir avec certitude l'existence d'une composante pré-berbère en Afrique du Nord.

\section{Des traces sociolinguistiques?}

En contact précoce avec toutes les grandes civilisations scripturaires et dominantes de la Méditerranée, l'Afrique du Nord est une région bien documentée et connue depuis longtemps. Or, ni les sources égyptiennes, ni les sources grecques (depuis Hérodote), ni les abondantes sources latines et arabes, ne font mention - en dehors des entités ethnoculturelles allogènes historiquement documentées - de la présence en Afrique du Nord d'un autre peuple et d'une autre langue indigènes que les Berbères et le berbère. Au contraire, toutes les sources anciennes sont unanimes et explicites (de Salluste à Ibn Khaldoûn en passant par St. Augustin): le peuplement et la langue autochtones de l'Afrique du Nord sont berbères. Il paraît assez improbable que tous ces observateurs et descripteurs aient pu laisser échapper l'existence, même résiduelle, d'une langue autre que le berbère.

\section{Des traces onomastiques?}

Le fond du matériau toponymique de l'Afrique du Nord est berbère, y compris, aujourd'hui, dans les régions les plus profondément arabisées. Les éléments nonberbères détectables sont quasiment toujours attribuables à une langue historiquement attestée (punique, latin, arabe et français, voire langues négro-africaines sur les franges sud du monde berbère). Dans le corpus toponymique, et cela à toutes époques, ce qui n'est pas clairement assignable à une langue allogène historiquement identifiée peut/ doit être rapporté au berbère, même si les étymologies et interprétations restent souvent obscures et problématiques. Même durant les périodes de très forte domination linguistique et culturelle allogène (périodes romaine et arabe notamment), derrière une couche ajoutée étrangère, on détecte facilement la nappe (substrat et adstrat) berbère, y compris dans le domaine de l'anthroponymie (Chaker 1984 et 1985 et EB XXXV, 2013, notice 017. 


\section{Des traces linguistiques (lexicales)?}

41 L'hypothèse d'une origine extérieure du berbère, quelle que soit la configuration précise retenue - à partir du Proche-Orient ou de l'Afrique de l'Est/sous la forme d'une arrivée significative et d'un renouvellement de population ou d'une diffusion plus perlée (diffusion par irradiation progressive défendue notamment par O. Durand 1993) -, suppose le maintien dans le lexique berbère, en particulier pour les réalités écologiques locales, d'un vocabulaire local "pré-berbère"; vocabulaire relicte qui, par définition, ne serait pas afro-asiatique. C'est sans aucun doute là l'une des fragilités les plus évidentes de toutes les thèses diffusionnistes concernant les origines berbères: personne n'a jamais pu cerner ce noyau lexical pré-berbère, qui permettrait d'étayer la thèse d'une "arrivée du proto-berbère » sur un substrat pré-berbère. Même ceux qui ont défendu ce genre de théories avec le plus de constance - comme Werner Vycichl $(1982,1983 \ldots)$, qui soutenait que le berbère était une langue "mixte" (grammaire protosémitique/lexique "méditerranéen ») - en restent au stade de la simple affirmation, sans apporter aucun élément concret de démonstration.

Or, il ne fait pas de doute que l'essentiel du stock de racines lexicales du berbère renvoie à des formes afro-asiatiques. On a montré dans un examen systématique de la structure de la racine et du système phonologique ( $C f$. Chaker 1995/b, chap. 16 et 2003a) que dans l'ensemble afro-asiatique la spécificité du lexique berbère est plus apparente que réelle. C'est aussi ce que montrent, à grande échelle, les reconstructions de Karl G. Prasse dans son Manuel de grammaire touarègue. Rien ne permet d'établir une origine non afro-asiatique du lexique berbère - dans sa composition globale ou, de manière plus spécifique, dans certains champs lexico-sémantiques - et, en tout cas, d'identifier un stock, même restreint, de formes lexicales que l'on pourrait assigner à un substrat non et pré-berbère identifié.

\section{Le substrat « Méditerranéen » (lexique et onomastique)}

Depuis longtemps ${ }^{11}$, on a signalé l'existence de matériaux lexicaux et onomastiques dont faire de diffusion couvre tout ou partie de l'espace méditerranéen, au sud (aire berbère) et au nord de la Méditerranée occidentale. Ainsi, des noms de fleuves ou cours d'eau fondés sur des séquences consonantiques $G R$ ou $S R / Z R$ se retrouvent aussi bien en Afrique du Nord qu'en Europe occidentale. Des ressemblances avec des matériaux ibériques, latins, grecs, sardes surtout, ont été signalées. Des noms de plantes spontanées se retrouvent également en latin, en grec... Par ex. : ifilku, "fougère" / latin filix, filica ; ikiker, "pois-chiche" / latin cicer; aliw, "olivier sauvage" / latin olea < grec ; tabuda, "massette, typha" / latin buda ; tayda, "pin" / latin taeda... L'origine de beaucoup de ces mots dans les langues indo-européenne est considérée par les spécialistes comme "inconnue", i.e. comme non indo-européenne.

On y a souvent vu la trace d'un substrat «méditerranéen ", pré-indoeuropéen, qui renverrait à une strate linguistique " euro-méditerranéenne ", voire « euro-africaine ", dans lequel la position du berbère reste ambiguë. L'origine de ces théories est ancienne et remonte à la fin du XIX et au début du XX $\mathrm{XX}^{\mathrm{e}}$ siècle avec des savants comme $\mathrm{H}$. Schuchardt qui ont attiré l'attention sur des faits de diffusion lexicale à très grande échelle, où le berbère et le basque sont souvent associés. Certains auteurs ultérieurs ont 
défendu l'idée d'une nappe linguistique ancienne centrée sur la Méditerranée, dont le basque et le berbère, mais aussi le corpus toponymique circum-méditerranéen, pourraient être les témoins, ou à tout le moins conserver des traces, et qui réunirait l'ensemble des langues pré-indo-européennes de la périphérie méditerranéenne et l'afro-asiatique (étendu au tchadique). H. G. Mukarovsky, l'un des auteurs les plus engagés dans cette voie, a utilisé des matériaux berbères et basques pour étayer la thèse d'un "euro-saharien".

Les bases linguistiques de ces tentatives de regroupements larges sont toujours très fragiles. Des critiques sévères ont été adressées aux tentatives de Mukarovsky par les chamito-sémitisants et les africanistes. Soumis à la critique du berbérisant, la très grande majorité des rapprochements avancés dans l'optique berbère/basque est à rejeter : là aussi, la critique étymologique préalable, interne au berbère, n'ayant pas été faite, on met le plus souvent en relation des lexèmes dont les ressemblances (signifiantes et signifiées) sont purement superficielles et secondaires.

Une certaine communauté des peuplements et des langues de l'Europe occidentale et du Nord de l'Afrique dans les temps paléolithiques ${ }^{12}$ n'est pas une idée absurde, mais les traces linguistiques en sont tellement ténues et problématiques qu'il sera, sans doute pour longtemps difficile, voire impossible, d'en administrer la preuve sur la base des méthodes admises de la linguistique historique et comparée. La question renvoie à des époques préhistoriques anciennes pour lesquelles la linguistique historique est peu armée et rencontre des limites probablement infranchissables ${ }^{13}$. Sur ce terrain, on en arrive vite aux convictions d'auteurs, même chez les spécialistes les plus érudits.

Quoiqu'il en soit de ces hypothèses «transcontinentales", anciennes ou récentes, le caractère non-indoeuropéen de ce petit stock lexical et toponymique est souvent bien établi. En revanche, la proposition symétrique est loin d'être établie : il n'est pas du tout démontré que ce matériel n'est pas berbère! En dehors des cas de diffusion lexicale avérés, la plupart des termes concernés peuvent recevoir une étymologie proprement berbère ou, à tout le moins, être rapprochés de racines ou formes berbères voisines par la forme et le sens, sans qu'il soit nécessaire de postuler un "mystérieux substrat méditerranéen pré-berbère » : c'est, par exemple, le cas des hydronymes de type GR, de tabuda/buda, de aliw/olea... (cf. Chaker, EB XXXIV, 2012, notice N58 b; EB XXXV, 2013, notice 016).

Pour ce qui est des plantes, arbres et arbustes, s'agissant de réalités endémiques en Afrique du Nord, l'hypothèse d'une origine berbère ne peut être a priori écartée. Les Berbères connaissaient ces végétaux bien avant les Indo-européens, qui n'en ont découvert la plupart qu'en arrivant sur les rives de la Méditerranée (au plus tôt à la fin du III ${ }^{\mathrm{e}}$ /début du II ${ }^{\mathrm{e}}$ millénaire avant J.C.). D'autre part, beaucoup de ces termes sont pan-berbères ou très largement attestés à travers l'espace berbère, et ont donc de fortes chances d'appartenir au fond lexical primitif de la langue. Dans quelques cas, des indices internes et externes plaident en faveur de leur origine berbère : ainsi tabuda, "massette, typha" (variété de roseau), terme pan-berbère, dénomme une plante sauvage omniprésente dans toute l'Afrique du Nord, qui a donné son nom à de nombreuses localités et lieux-dits dans tout l'espace berbère, depuis l'Antiquité; et la séquence consonantique $B D$ peut immédiatement être rattachée à la racine lexicale pan-berbère $B D(D)$, "être debout, se dresser" dont le signifié paraît tout à fait compatible avec celui du végétal qui se dresse, comme la massette ou le roseau. La probabilité d'une origine berbère est presque aussi forte pour aliw (cf. Chaker, EB XXXV, 
2013, notice 016)... Quant aux hydronymes sur base $G R, \gamma R, S R$..., leur lien avec des racines berbères, voire afro-asiatiques, n'est pas moins probable (cf. Chaker, EB XXXIV, 2012, notice N58b).

49 En définitive, en dehors de cas comme berb. asnus / lat. asinus qui peuvent effectivement provenir d'une troisième langue asiatique ${ }^{14}$, l'hypothèse de l'emprunt au berbère par les langues pré-indoeuropéennes puis indoeuropéennes (latin, grec, sarde...) du Nord de la Méditerranée, si elle est difficile à établir, n'est pas du tout irréaliste et apparaît même comme la plus vraisemblable.

Dans le cas du berbère, le recours à un «substrat méditerranéen " revient en fait à tenter d'expliquer l'obscur par l'inconnu et à sous-estimer l'importance des phénomènes de diffusion lexicale. Rien n'interdit d'envisager que ces termes, berbères (ou proto-berbères), se soient largement diffusés sur les pourtours de la Méditerranée occidentale, avant l'arrivée de Indo-européens: l'Afrique du Nord est en relation d'échange avec tout l'arc nord-méditerranéen (Péninsule ibérique, Sicile, Italie, mer Égée...) dès les temps paléolithiques et tout au long du Néolithique et de la protohistoire ${ }^{15}$.

\section{Le vocabulaire fondamental de l'agriculture, de l'élevage et des techniques connexes, permet-il de déceler une origine extérieure ?}

On a pu montrer (Chaker 1995/a, 1996 et EB XXXIV, 2012, notice N49) que l'essentiel du vocabulaire des animaux domestique et des céréales est à la fois proprement berbère et pan-berbère, ce qui est un indice de son ancienneté et de sa genèse locale. Cela va aussi plutôt dans le sens d'une néolithisation endogène. Le vocabulaire des paramètres centraux de la néolithisation (l'élevage et la céréaliculture) est spécifiquement berbère et ne permet pas de détecter une expansion venue d'ailleurs, fondée sur la « révolution néolithique ", qui aurait permis à des populations proche-orientales ou est-africaines d'imposer, brutalement ou progressivement, leur langue.

On peut même, dans quelques cas favorables (mil/sorgho, jujubier/figue/raisin...), mettre en évidence une sémanto-genèse indigène, à partir de signifiés nécessairement pré-néolithiques. L'étude lexico-sémantique de certaines racines berbères fondamentales dans le domaine de l'agriculture (Chaker 1997/b et EB XVIII, F23 ; XXVI, J19 ; XXXII, Ml $16 \mathrm{~b}$; XXXIV, N49) permet de montrer que le signifié agricole actuel s'est probablement formé de manière interne au berbère, à partir de significations plus anciennes, antérieures à l'agriculture, car ces racines ont d'abord désigné des réalités non agricoles, voire même non végétales.

\section{Le système grammatical et le matériel morphologique du berbère va-t-il dans le sens d'une arrivée extérieure?}

L'extraordinaire unité du système grammatical du berbère, sur une aire géographique immense, ne va pas dans le sens de la thèse d'une origine extérieure récente de la langue, qui supposerait une diffusion, nécessairement lente et aléatoire dans ses résultats, sur un (des) substrat(s) pré-berbère(s).

54 En effet, le système grammatical et le matériel morphologique du berbère présentent dans l'ensemble afro-asiatique une cohérence, une simplicité et une transparence, qui 
permettent souvent de relier le berbère actuel aux formes les plus anciennement attestées ou reconstruites de l'afro-asiatique (notamment à l'akkadien pour le système verbal). On renverra ici aux travaux sur les traits afro-asiatiques du berbère (Zaborski 1984 ; Chaker, EB VI, 1989, notice A247; Chaker 1995b, chap. 16), qui confirment non seulement que le berbère est bien une langue afro-asiatique, mais plus encore qu'il en présente souvent des caractéristiques archaïsantes et très " pures "; par de nombreux aspects centraux (le système verbal, la dérivation verbo-nominale, le système pronominal notamment), le berbère est bien au centre et non dans une périphérie évoluée et recomposée de l'afro-asiatique. Une telle configuration suppose une grande stabilité, linguistique et sociolinguistique, peu compatible avec la thèse d'une arrivée extérieure et/ou d'une diffusion progressive sur un substrat pré-/non-afro-asiatique.

$55 \mathrm{Au}$ sein du berbère, les divergences systémiques lourdes, i.e. non-explicables par des évolutions régionales récentes à partir d'un système commun, sont extrêmement rares et concernent, pour la plupart, le touareg qui, contrairement à l'opinion répandue, pourrait bien être une branche périphérique du berbère, fortement influencée par des substrats/adstrats négro-africains ${ }^{16}$. On notera, par exemple, que le système vocalique du touareg, si particulier au sein de l'ensemble berbère, se superpose quasiment parfaitement à celui du haoussa (cf. Newman 2000, p. 398). Alors qu'il est impossible de repérer une divergence systémique ancienne entre le kabyle (Algérie centrale) et le tachelhit (sud-ouest du Maroc).

Le système grammatical du berbère montre une identité afro-asiatique très marquée et un lien particulièrement étroit avec le sémitique ${ }^{17}$ - et ne manifeste, dans son ensemble, aucune trace de recomposition, créolisation ou de mélanges de systèmes qui appartiendraient à des phylums distincts. Là encore, comme pour le niveau lexical, il ne paraît pas possible de mettre en évidence, sur la base d'arguments concrets, un quelconque «brassage de systèmes». On est bien dans l'unité, la cohérence et la continuité linguistique.

\section{Une unité et stabilité linguistiques étonnantes : hypothèses explicatives}

Cette stabilité remarquable, sur un territoire immense, du système linguistique berbère, dans toutes ses composantes essentielles, est surprenante, voire déroutante. Parmi les langues et familles de langues, elle apparaît exceptionnelle, surtout quand on la compare avec des domaines voisins qui ont connu des reconfigurations, restructurations très profondes, souvent en des laps de temps relativement courts : de l'ordre de cinq millénaires pour le domaine indo-européen, à peine un millénaire pour les langues romanes...

\section{Une langue à évolution lente?}

58 Stabilité également très marquée à travers le temps, si l'on considère les éléments, rares mais nets, que nous apportent les sources anciennes médiévales ou antiques (libyques). Les formes médiévales les plus anciennes de berbère accessibles - ce ne sont souvent que des bribes (El-Bekri, documents almohades, ibadites...) - sont quasiment du "berbère contemporain ", bien qu'elles soient âgées pour certaines de près d'un millénaire. Elles comportent certes des obscurités lexico-sémantiques, bien naturelles, mais leur grammaire est déjà celle du berbère. L'étude des matériaux libyques, notamment de la composante onomastique (cf. notice 017 «Onomastique libyco- 
berbère »), conduit aussi à conclure qu'une partie de la grammaire du verbe*, et probablement celle du nom*, du berbère moderne est déjà en place à époque antique. Tout, certes, n'est pas transparent dans le matériel linguistique libyque - loin s'en faut !-, mais les éléments interprétables sont tous clairement déjà "berbères ", plus exactement ils sont conformes aux structures élémentaires de la langue berbère et s'interprètent, parfois très aisément, à partir des données lexico-sémantiques berbères: on renverra à des exemples comme: YRN (уərПA) / YRNTN (yərna-tən)/ TRNTN (Tərna-tən); YDR (Yidir)... qui n'autorisent aucune discussion quant à leur "berbérité ", et pourtant ils ont plus de 2.000 ans!

On insistera lourdement sur ce point : même si les données dont nous disposons sont très lacunaires, fragmentaires, c'est une donnée non-contestable que des éléments de paradigmes grammaticaux fondamentaux (indices de personnes, affixes personnels, marques nominales, prépositions...), des racines lexicales sont déjà attestés dès l'antiquité quasiment sous leur forme berbère actuelle. A ce constat initial, on rajoutera que ces éléments sont souvent présents dans des matériaux onomastiques; or ce type de matériel est volontiers conservateur. Autrement dit, des anthroponymes ou des toponymes antiques, voire protohistoriques, parfaitement "berbères ", comme YRN / YRN-TN / YDR, Thizi ou Thala... illustrent nécessairement une grammaire déjà en place depuis longtemps. Ce qui assoit d'autant plus la thèse d'une grande stabilité de la langue à travers le temps. Cette idée n'est d'ailleurs pas nouvelle: on la trouve, au moins de manière implicite, dans la pensée d'André Basset qui écrivait :

«... Cette langue expression d'un société à l'évolution des plus lentes, garde une

large part de faits communs à l'ensemble du groupe et, partant, identiques ou peu s'en faut d'un bout à l'autre du domaine. » (1952/1969, p. 2).

60 Il n'est bien sûr pas question de prétendre que le berbère n'a pas connu d'évolution depuis 2000 ans - certains changements sont très précisément détectables à travers la comparaison interdialectale ou les sources médiévales -, ni de réhabiliter les conceptions essentialistes du $\mathrm{XIX}^{\mathrm{e}}$ sur "l'esprit des langues", ou les thèses sur "l'immutabilité des langues sémitiques" théorisées par Renan (1858, notamment p. 424-5), mais les constats précédents obligent à admettre que le berbère a connu au cours de son histoire une évolution particulièrement lente.

61 Non seulement les structures élémentaires de la langue et l'essentiel de son stock lexical sont communs à l'ensemble de faire berbère contemporaine, mais la langue semble aussi avoir assez peu évolué depuis deux, voire trois millénaires. Plusieurs auteurs ont récemment essayé d'apporter des éléments d'explication de cette situation singulière.

Blench (2001) a proposé d'y voir l'impact d'un pastoralisme nomade préhistorique généralisé qui, par la régularité des échanges aurait contribué à l'homogénéité de la langue. L'idée est séduisante et semble être confirmée par les données de l'archéologie préhistorique qui établissent nettement que le néolithique nord-africain est d'abord lié à la domestication animale et à l'élevage pastoral et non à l'agriculture (cf. les différentes notices "Néolithisation", notamment: N40, N45). Elle pourrait aussi trouver un écho dans les données lexico-sémantiques berbères : on a montré qu'à partir du lexique actuel, il n'est pas possible de dégager une dénomination berbère commune de la maison* (habitation des humains en dur; cf. notice M17c). De même, le vocabulaire de l'outre* présente une grande unité, qui traverse milieux nomades et 
sédentaires, ce qui pourrait aussi être la trace d'un pastoralisme nomade ancien quasi généralisé (cf. notice 058).

Par ailleurs, l'histoire politique et ethnique de l'Afrique du Nord, à toutes époques, fournit des indices très nombreux d'une grande mobilité des populations, sur des distances considérables : les tribus se déplacent au cours des temps - d'Est en Ouest, du Nord au Sud - sur des centaines, voire des milliers de kilomètres, nouent des alliances à grande distance; les chefs de révoltes et de guerres, de l'antiquité ou de la période médiévale, lèvent des troupes, trouvent refuge dans des régions/auprès de populations éloignées... Même dans des régions telliennes de tradition sédentaire actuellement très affirmée comme la Kabylie, on constate que :

a. Sur une profondeur de quelques siècles, la tradition orale fait presque toujours venir les familles et les groupes plus larges d'un "ailleurs», parfois éloigné et souvent assez précisément documenté ${ }^{18}$;

b. L'architecture traditionnelle est extrêmement légère et éphémère et ne donne jamais naissance à des constructions imposantes et/ou durables, sauf bien entendu cas d'influence citadine notoire ;

c. Le mobilier traditionnel est lui aussi, pour l'essentiel, léger voire sommaire et ne se différencie guère de celui que l'on pouvait rencontrer chez les groupes nomades ou seminomades. Les imposants coffres anciens (asənduq, afniq; cf. Gast \& Assié 1993) sont la seule exception notable à cette règle pour la Kabylie, mais ces meubles devaient être assez rares et certainement réservés à quelques familles de notables aisés.

Tout milite en faveur d'une tradition pastorale nomade et semi-nomade dominante, et en tout état de cause, une grande mobilité des groupes humains, jusqu'à une époque tardive (fin des temps médiévaux).

Louali \& Philippson (2004) ont avancé une autre hypothèse explicative : la forte unité $\mathrm{du}$ berbère ne serait pas ancienne (préhistorique) mais protohistorique et serait la conséquence d'un processus secondaire d'homogénéisation linguistique, qu'ils proposent d'identifier comme un "proto-berbère 2 ", et qu'ils attribuent à une influence de/réaction à la présence punique et à l'action des royaumes libyques protohistoriques et antiques. La thèse est moins convaincante: la nature des formations politiques antiques est bien connue (Camps 1961) et parait incompatible avec une action profonde d'unification linguistique et culturelle. Comme le rappellent Laporte \& Ghaki (notice N76 «Numide, Numidie»), les formations politiques protohistoriques et antiques libyques «ne revêtaient pas la forme de monarchies absolues, mais devaient s'appuyer d'une part sur des villes relativement autonomes, et d'autre part sur des allégeances tribales parfois fragiles». Les Etats libyques protohistoriques et antiques tiennent tous leur pouvoir de leurs assises tribales et n'ont jamais disposé d'un appareil d'état, d'un contrôle du territoire, d'institutions culturelles qui auraient pu permettre une unification autour d'un " centre ", numide ou autre. Tout indique que, même au moment du rayonnement maximal de la dynastie numide (sous le règne Massinissa*), le monde libyque restait une société fragmentée, tribale, diverse et, surtout, sans institutions culturelles communes unifiées, religieuses ou de quelque autre nature.

En fait, si l'on devait envisager une dynamique d'homogénéisation secondaire du berbère, on devrait plutôt la situer aux $\mathrm{V}^{\mathrm{e}}$-VII ${ }^{\mathrm{e}}$ siècle, après l'effondrement de l'administration romaine, qui a été suivie par une " poussée berbère ", et probablement une reberbérisation (totale ou partielle) des plaines et des villes jusque là largement 
acquises à la langue et à la culture latines. Mais, en tout état de cause, un tel phénomène aurait touché des zones malgré tout limitées de l'espace berbère et ne permet pas d'expliquer l'unité linguistique globale.

On retiendra plutôt la thèse d'une homogénéité linguistique préhistorique (au moins épipaléolithique) du territoire, confortée, avec la néolithisation, par une économie pastorale généralisée - comme le suggère Blench et le confirment les préhistoriens du néolithique -, perdurant jusqu'aux temps modernes. Mais d'autres paramètres peuvent avoir joué un rôle important dans cette stabilité linguistique à travers l'espace et le temps. On proposera notamment de prendre en considération deux autres traits qui pourraient avoir été décisifs :

- La faiblesse de la démographie qui, conjuguée avec une grande mobilité des groupes humains, permettrait de comprendre à la fois l'unité marquée de la langue et en même temps les enchevêtrements d'isoglosses, même sur des points non-marginaux de la structure linguistique.

- Une vitesse d'évolution exceptionnellement lente de la langue berbère. Ce trait, qui mérite évidemment des recherches et approfondissements ultérieurs, serait déterminé à la fois par les caractéristiques sociolinguistiques évoquées ci-dessus (homogénéité linguistique du territoire, échanges permanents/mobilité des populations) et des traits de structure de la langue : existence d'un marquage morphologique lourd du verbe et du nom, existence d'un système de dérivation verbo-nominale à la fois transparent et relativement simple, stock de morphèmes grammaticaux simples et très polyvalents...

\section{Conclusion}

Du rappel des données préhistoriques acquises et de l'examen des matériaux linguistiques, on tire, si ce n'est une démonstration, du moins le sentiment fort qu'il n'existe aucun argument décisif en faveur d'une origine extérieure globale - moyenorientale ou africaine - des Berbères et/ou de leur langue. Au contraire, tous les indices vont dans le sens d'une très grande stabilité et d'une continuité du peuplement et de la langue berbère dans son aire d'extension actuelle, dont les limites ont peu changé depuis des millénaires. Le seul mouvement de population bien établi est l'extension progressive vers le sud (Sahara et Sahel), amorcée depuis le IV e millénaire avant J.-C. ; donnée qui conforte, incidemment, notre hypothèse selon laquelle le touareg est par certains aspects une forme "périphérique » évoluée du berbère, ayant subi l'influence de substrats/adstrats négro-africains.

En définitive, tout laisse à penser que les thèses d'une origine extérieure du peuplement et de la langue berbères reposent toutes et toujours sur un postulat implicite : les Berbères, «dernière grande réserve de Barbares méditerranéens », leur langue, sans solide tradition écrite et jamais normalisée, ne peuvent être qu'un rejeton périphérique d'un " centre » plus consistant, plus sérieux », le Moyen-Orient, berceau de la civilisation, ou l'Afrique de l'Est, berceau de l'humanité...

Mais si l'on considère objectivement les données et équilibres globaux de l'ensemble afro-asiatique, force est de constater que le seul espace de continuité et de stabilité linguistique, sur la très longue durée et d'une certaine ampleur géographique, est bien le monde berbère. C'est là un fait concret, peu discutable, qui est de nature à amener à reconsidérer totalement la question du berceau primitif de cette famille de langues. 
71 On n'est donc pas loin de penser que si l'on doit chercher un môle de stabilité humaine qui puisse être le berceau primitif de l'afro-asiatique, on aurait quelques chances de le trouver en Afrique du Nord.

Par ailleurs, la proximité très marquée du berbère avec la branche sémitique, dont elle n'est géographiquement séparée que par l'étroite bande de la vallée du Nil occupée par l'égyptien, autorise à poser l'existence, au sein de l'afro-asiatique, d'un sous-ensemble berbéro-sémitique " méditerranéen », qui a pu irradier vers l'Ouest (Moyen-Orient) et le sud (Afrique centrale et orientale).

73 Tout montre en tout cas que les Berbères et leur langue ont des racines fort anciennes en Afrique du Nord et qu'il n'existe aucune donnée certaine qui autoriserait à les considérer comme venant d'ailleurs, ni eux, ni leur langue, du moins à l'échelle des temps néolithiques et paléolithiques récents.

$74 \rightarrow$ Voir aussi "Apparentement" (A247), EB VI, 1989 et dossier "Néolithisation Néolithique" (N40 à N49), EB XXXIV, 2012.

\section{BIBLIOGRAPHIE}

BASSET A., 1952 (1969) - La langue berbère, Londres.

BASSET A., 1957 - Articles de dialectologie berbère, Paris, Klincksieck.

BATES O., 1914 - The Eastern Libyans, Londres [réédition 1970)

BEHRENS P., 1984 - "Wanderungsbewegungen und Sprache der früheren saharanischen

Viehzüchter", Sprache und Geschichte in Afrika, 6, 1984-85.

BERTOLDI V., 1931 - «Problèmes de substrat », BSLP, XXXII, p. 93-183. Linguistica storica, Napoli.

BERTOLDI V., 1943 - Linguistica storica, Napoli.

BERTOLDI V., 1950 - Colonizzazioni dell'antico Mediterraneo occidentale alla luce degli aspetti linguistici, Napoli.

BERTOLDI V., 1952 - Progressi di metodo nella ricerca etimologica con particolare riguardo ai mediterranismi attestati dal greco al latino, Napoli.

BLENCH R., 2001 - "Types of language spread and their archeological correlates : the example Berbers", Origini. Preistoria e Protoistorai delle Civiltà Antiche, XXIII, p. 169-189.

BYNON J., 1985 - "Berber and Chadic : the lexical evidence", Current progress in Afro-Asiatic Linguistics, third international hamito-semitic congress.

CAMPS G., 1961 - Massinissa ou les débuts de l'Histoire, Alger, Imprimerie officielle.

CAMPS G., 1974 - Les civilisations préhistoriques de l'Afrique du Nord et du Sahara, Paris, Doin.

CAMPS G., 1980 - Berbères. Aux marges de l'histoire, Toulouse, Edit. des Héspérides. Réédition sous le titre : Berbères. Mémoire et identité, Paris, Editions Errances, 1987 et Arles, Actes Sud (Babel), 2007. 
CAMPS G., 1981 - « L'origine des Berbères », Islam, société et communauté. Anthropologie du Maghreb, Paris, Editions du CNRS (« Les Cahiers du CRESM », 12), p. 9-33.

CAMPS G., 1982 - Introduction à la préhistoire. A la recherche du paradis perdu, Paris, Librairie académique Perrin.

CHAKER S., 1984 - Textes en linguistique berbère (introduction au domaine berbère), Paris, Cnrs.

CHAKER S., 1985 - « Onomastique berbère ancienne (Antiquité/Moyen âge) : rupture et continuité ", ВСтн ( $2^{\circ}$ Congrès d'Histoire et d'Archéologie de l'Afrique du Nord), n.s., 19, 1983, p. 483-497. CHAKER S., 1989 - « Apparentement (de la langue berbère) », Encyclopédie berbère, VI, p. 812-820. CHAKER S., 1995/a - «Linguistique et préhistoire : autour de quelques noms d'animaux domestiques en berbère ", L'homme méditerranéen. Mélanges offerts à Gabriel Camps, Publications de l'Université de Provence, Aix, p. 259-264.

CHAKER S., 1995/b - Linguistique berbère. Etudes de syntaxe et de diachronie, Paris/Louvain, Peeters, 275 p. Notamment chap. 15 : «Comparatisme et reconstruction. Domaine chamito-sémitique » et chap. 16 : «La parenté chamito-sémitique du berbère : un faisceau d'indices convergents ».

CHAKER S., 1997/a - "Quelques faits de grammaticalisation dans le système verbal berbère ", Mémoires de la Société de Linguistique de Paris, n.s., V, 1997 (« Grammaticalisation et reconstruction »), p. 103-121.

CHAKER S., 1997/b - « Figuier/figues (nom berbère) », Encyclopédie berbère, XVIII, p. 2831-283.

CHAKER S., 2001 - « Indices de personne », Encyclopédie berbère, XXIV, p. 3725-3729.

CHAKER S., 2003a - « Autour de la racine en berbère », Folia Orientalia (Cracovie), 39, p. 83-93.

CHAKER S., 2003b - « Résistance et ouverture à l'Autre : le berbère, une langue vivante à la croisée des échanges méditerranéens. Un parcours lexicologique. ", Actes du colloque L'interpénétration des cultures dans la bassin occidental de la Méditerranée (Paris, Sorbonne, 14/11.2001), Paris, Mémoire de la Méditerranée, 2003, p. 131-154.

CHAKER S., 2005 - « Les paradigmes personnels du berbère ", Systèmes de marques personnelles en Afrique (édité, par. D. Ibriszimow et G. Segerer), Louvain/Paris, Peeters (“Afrique et Langage”, 8), 2004, p. 43-54.

CHAKER S., 2006 - « Aux origines berbères : préhistoire et linguistique. Allochtonie/autochtonie du peuplement et de la langue berbères ? », Faits de Langues, 27 (" Les Langues chamito-sémitiques »), vol. 2, p. 235-244.

COHEN D., 1968 - « Les langues chamito-sémitiques », Le langage, Paris, NRF-Gallimard ("La Pléïade").

COHEN D., 1983 - «Qu'est-ce qu'une langue sémitique », GLECS, XVIII-XXIII, 1973-1979 (3).

DIAKONOFF I. M., 1965/1988 - Semito-hamitic languages, Moscou. Réédition révisée : 1988, Afrasian languages, Moscou, Nauka.

DURAND O., 1993 - «Qu'est-ce qu'une langue berbère ? Hypothèses diachroniques ", RENDICONTI (Atti della Accademia Nazionale dei Lincei) : IX/IV (1).

EHRET Chr., 1995 - "Reconstructing Proto-Afroasiatic (Proto-Afrasian) : Vowels, Tones, Consonants and Vocabulary", Linguistics, 126 (University of California Publications). 
EHRET Chr., 1995 - "Who were the rock painters ? Linguistic evidence for the Holocene populations of the Sahara, News 95", Proceedings of the International Rock Art Congress (30 august, 6 september 1995, Pinerolo), p. 96-97.

Encyclopédie berbère, Aix-en-Provence, EDISUD, $1984 \rightarrow$ (Dir. : G. Camps, puis S. Chaker).

GALAND L., 1977 - «Continuité et renouvellement d'un système verbal : le cas du berbère », BSLP, LXXII/1.

GALAND L., 1983 - « Berbère et traits sémitiques communs », GLECS, XVIII-XXIII, 1973-79 (3).

GAST M. \& ASSIÉ Y., 1993 - Des coffres puniques aux coffres kabyles, en collaboration avec Yvette Assié, Paris, CNRS Editions, 254 p.

GREENBERG J., 1955 - Studies in African Linguistic Classification, New Haven.

GREENBERG J., 1966 (1963), Languages of Africa, The Hague, Mouton.

GSELL St., 1918-1928 - Histoire ancienne de l'Afrique du Nord, Paris, [t. V, 1925].

HACHI S., 1999 - «L'Homme de Méchta-Afalou, Cro-Magnon de l'Afrique du Nord durant les 20 derniers millénaires : évolution culturelle et devenir ». HDR, Université Paul Valéry, Montpellier III, 4 volumes. HACHI S. et alii, 2002 - « Figurines du Paléolithique supérieur en matière minérale plastique cuite d'Afalou Bou Rhummel (Babors, Algérie) ». Premières analyses par spectroscopie Infrarouge. L'Anthropologie, 106, p. 57-97.

HACHI S., 2003 (a) - Les cultures de l'Homme de Mechta-Afalou. Le gisement d'Afalou Bou Rhummel (massif des Babors, Algérie). Les niveaux supérieurs 13 000-11 000 BP., CNRPAH, Alger.

HACHI S., 2003 (b) - Aux origines des Arts premiers en Algérie. Les figurines et les objets modelés en terre cuite de l'Abri-sous-roche préhistorique d'Afalou, Babors, Algérie, 18 000-11 000 BP. CNRPAH, Alger. HACHID M., 2000 - Les premiers Berbères, Aix-en-Provence/Alger, EDISUD/Inayas.

HUBSCHMID J., 1953 - Sardische Studien. Das mediterrane Substrat des Sardischen, seine Beziehungen zum Berberischen und Baskischen sowie zum eurafricanischen und hispano-kaukasischen Substrat der romanischen Sprachen, Romanica Helvetica, 41 Bern

HUBSCHMID J., 1960 - Mediterrane Substrat, Bern, Francke.

HUBSCHMID J., 1968 - « Sprachgeographie und Substratforschung », Festschrift Walther von Wartburg zum 80. Geburtstag, II, Tubingen, p. 8-12

HUBSCHMID D., 2008 - « Sostrati mediterranei rivisitati », in Aspesi F., Brugnatelli V., Callous A. L., Rosenzweig C. (eds) : Il mio cuore è a Oriente. Studi di linguistica storica, filologia e cultura ebraica dedicati a Maria Luisa Mayer Modena, Milano.

IBN KHALDOUN, 1925 - Histoire des Berbères, Paris (rééd.).

JUCQUOIS J., 2009 - «La linguistique génétique revisitée : la controverse du protomondial», La Linguistique, 45/1, p. 133-150.

LEFEVRE-WITIER Ph., 2002 - «Berbérophones : génétique et langage ", Journal des anthropologues, $\mathrm{n}^{\circ}$ 90-91, p. 401-414

LOUALI N. \& PHILIPPSON G., 2004 - "Berber Expansion into and within northwest Africa : a linguistic contribution", Africa und Übersee, 87, p. 105-130.

MILITAREV A. \& SHNIRELMAN V. A., 1988 - "The Problem of Location of Early Afrasians...", 12th International Congress of Anthropological and Ethnological Sciences, Zagreb 1988, Moscou, Nauka. 
MOSCATI S. et al., 1964 - An Introduction to the comparative grammar of the Semitic Languages, Wiesbaden.

MUKAROVSKY H. G., 1964 - “Baskisch und Berberisch”, Wiener Zeit-schrift für die Kunde der Morgenländer, 59/60.

MUKAROVSKY H. G., 1966 - « Les rapports du basque et du berbère », GLECS, X.

MUKAROVSKY H. G., 1966-67 - « Les langues apparentées au chamito-sémitique », GLECS, XI.

MUKAROVSKY H. G., 1966-67 - « L'Euro-euskarien et les langues ouest-africaines », GLECS, XI.

MUKAROVSKY H. G., 1981 - "Hamito-semitisch, Afro-asiatisch, Erythräisch : zum Wandel von Begriffen und Verstandnis", Zeitschrift für Phonetik..., 34/5.

NEWMAN P., 2000 - The Hausa Language : An Encyclopedic Reference Grammar, New Haven, Yale University Press.

PENCHOEN TH. G., 1968 - « La glottochronologie », Le langage, Paris, NRF-Gallimard (La Pléïade).

PRASSE K.-G., 1972-74 - Manuel de grammaire touarègue (tahaggart), Copenhague, Akademisk Forlag, 1972 : I-III, Phonétique-Ecriture-Pronom, 1974 : IV-V, Nom ; 1973 : VI-VII, Verbe ; 2009 : VIII-IX, Syntaxe (Schwülper, Cargo Verlag).

PRÉHISTOIRE MAGHRÉBINE : Actes du colloque international de Tamanrasset (5-7 novembre 2007), Alger, Cnrpah (Travaux du Cnrpah, n.s. : 11), 2011, t. I, 336 p. ; t. II, 325 p.

RENAN E., 1858 - Histoire et système comparé des langues sémitiques, Paris, Imprimerie Impériale, $2^{\mathrm{e}}$ édition.

ROUBET C., HACHI S., F. KHERBOUCHE, 2011 - « Vivre ensemble depuis 20000 ans au moins en Afrique du Nord : expression d'une communication », in A. Mettouchi (ed), Parcours berbères : Mélanges offerts à Paulette Galand-Pernet et Lionel Galand. Köln : Köppe (Berber Studies n 33).

RÖSSLER O., 1952 - "Der semitische Charakter der libyschen Sprache", Zeitschrift für Assyriologie..., n.F., 16.

RÖSSLER O., 1964 - "Libysch-Hamitisch-Semidsch”, Oriens, 17.

RÖSSLER O., 1979 - “Berberisch-Tschadisch Kernvokabular”, Africana Marburgensia, 12/1-2.

RUHLEN M., 1997 - L'origine des langues. Sur les traces de la langue mère, Paris, Belin.

SCHUCHARDT H., 1913 - Baskisch und Hamitisch, Paris, 1913.

SIMON-NAHUM P., 2001 - «Renan et l'histoire des langues sémitiques », Histoire - Epistémologie Langage, 23/2, p. 59-75.

TERRACINI B., 1927 - « Osservazioni sugli strati più antichi della toponomastica sarda », Convegno archeologico Sardo, giugno 1926, Reggio Emilia, p. 137-150

TERRACINI B., 1936 - « Gli studi linguistici sulla Sardegna preromana, Sardegna Romana, Roma p. 110-135.

TILlET T. (éd.), 1997 - Sahara. Paléomilieux et peuplement préhistorique au Pléistocène supérieur, Paris, L'Harmattan.

TILLET T., (à paraître) -« L'Atérien du Sahara méridional », Hommage à Marceau Gast..., (S. Chaker \& H. Claudot, éds.), Louvain/Paris, Peeters. 
VYCICHL W., 1981 - « Problèmes de linguistique chamitiques : morphologie et vocabulaire », GLECS, XVIII-XXIII, 1973-79 (3).

VYCICHL W., 1983 - «Contacts chamito-sémitique : un seul groupe ou deux groupes distincts? », GLECS, XVIII-XXIII, 1973-79 (3).

VYCICHL W., 1984 - « Linguistica comparativa camito-semitica », Atti della terza giornata di studi camito-semitici e indoeuropei (= Studi Semitici, n. s. 1), Rome.

VYCICHL W., 1987 - «The Origin of the Hamito-Semitic Languages », Proceedings of $4^{\text {th }}$ International Hamito-Semitic Congress (Hamburg, 1983), H. Junraith-mayr \& W. Müller, eds.), Amsterdam/ Philadelphia, John Benjamins Publishing Company (Current Issues in Linguistic Theory : 44), p. 109-121.

WAGNER M. L., 1907 - « Gli elementi del lessico sardo », Archivio Storico Sardo III, p. 370-420,

WAGNER M. L., 1931 - « Über die vorrömischen Bestandteile des Sardischen », Archivum Romanicum $X V$, p.207-247

WAGNER M. L., 1933 - « Osservazioni sui sostrati etnico-linguistici sardi », Revue de Linguitique

Romane IX, p. 275-284

WAGNER M. L., 1943 - « zum Paläosardischen », Vox Romanica IX, p. 306-323

WAGNER M. L., 1997 [1951] - La lingua sarda. Storia, spirito e forma, Bern, Francke. Nouvelle edition (a cura di G. Paulis) : Nuoro, Ilisso, 1997.

WILLMS A., 1968 - "Inwieweit kann die Berbersprachforschung der hamito-semitischen Sprachvergleichung dienen?", Islam, 44, 1968.

ZABORSKI A., 1984 - "The Stages of Hamito-semitic", Rocznik Orientalistyczny, 43.

ZABORSKI A., 1988 - “Zum hamitosemitischen Charakter des Berberischen”, Progressive traditions in african and oriental studies, Berlin, Akademie Verlag.

\section{NOTES}

1. La remarquable synthèse collective que constituent les «Actes du colloque international de Tamanrasset: 5-7 novembre $2007 »$ : Préhistoire maghrébine, Alger, CNRPAH, 2011, confirme clairement ces liens étroits avec l'Europe du sud; cf. notamment les contributions de Muñoz, Otte, Coppa et al.

2. Les ouvrages de référence de G. Camps $(1974,1982 . .$.$) , sur bien des points, sont eux-mêmes$ largement dépassés : sur la question de l'art préhistorique (qui, pour lui, était absent dans l'Ibéromaurusien et était d'origine capsienne); sur le caractère non-maritime du Capsien... On évoquera aussi, à un niveau plus global, le renouvellement constant des datations et des filiations à l'intérieur du genre Homo, ou le débat sur le statut (espèces distinctes ?) des Néandertaliens et des Cro-Magnon...

3. On lira sur ce point avec intérêt l'étude «Berbérophones : génétique et langues » de notre regretté collègue $\mathrm{Ph}$. Lefèvre-Witier (2002), qui montre bien la labilité des caractéristiques génétiques au sein des groupes et les fragilités méthodologiques des recherches sur le sujet.

4. De nombreuses facettes en sont présentées dans les Actes du colloque international de Tamanrasset : Préhistoire maghrébine, Alger, CNRPAH, 2011. 
5. Les préhistoriens nous pardonneront de parler ici et dans la suite de ce texte, par pure commodité terminologique, de "Capsiens" et "Ibéromaurusiens" pour désigner ces populations alors que ces deux termes, stricto sensu, n'identifient que des cultures.

6. Préhistoire maghrébine, t. I, 2011 : cf. Kefi-Ben Atig \& Béraud-Colomb.

7. Sans parler des indices génétiques, objet encore de nombreuses incertitudes et débats; mais les recherches récentes semblent bien indiquer un hiatus anthropologique net entre Protoméditerranéens nord-africains et Natoufiens et une plus grande affinité avec le peuplement européen (Préhistoire du Maghreb, t. I : cf. Copa et al.

8. Corroborées par les découvertes dans le Rif oriental (Taforalt), cf. M. Nami (Préhistoire du Maghreb, 2011, t. I).

9. Au paléolithique moyen et supérieur, la culture atérienne, originaire de l'Afrique du Nord, va progressivement s'étendre à tout le Sahara et aux régions sahéliennes de l'Afrique de l'Ouest, jusqu'à la côte atlantique... Ce qui rappelle opportunément que l'Afrique du Nord n'a pas toujours été qu'un point d'arrivée de dynamiques culturelles et/ou humaines exogènes, elle a aussi pu être un point de départ.

10. On peut se demander s'il ne s'agit pas là d'un effet de censure du " politiquement correct »! Mais lorsqu'une hypothèse est formulée, il est bon d'en vérifier toutes les conditions de possibilité et les implications.

11. Voir les travaux de Bertoldi, Hubschmid, Terracini, Wagner...

12. Hypothèse qui semble corroborée par les données de l'anthropologie préhistorique (Préhistoire du Maghreb, t. I, 2011, cf. notamment Copa et al.)

13. On trouvera une solide mise au point récente sur les problèmes et limites des reconstructions linguistiques sur des périodes de temps très longues sous la plume du linguiste indo-européaniste Jacques Jucquois (2009).

14. Au moins l'une des variétés de l'âne nord-africain est d'origine asiatique (cf. Camps 1988).

15. Préhistoire maghrébine: Actes du colloque de Tamanrasset, I, 2011, cf. les contributions de J. R. Munoz, M. Otte...

16. Le cas du zenaga de Mauritanie est encore plus évident sur ce plan des probables influences négro-africaines.

17. On rappellera que, dès 1952, O. Rössler avait affirmé le « caractère sémitique du berberbère» (même s'il semble avoir ultérieurement abandonné cette position). En tout cas, les convergences structurales, notamment grammaticales, sont extrêmement fortes entre les deux branches.

18. En Kabylie, quasiment toutes les enquêtes et monographies villageoises aboutissent au même constat : toutes les composantes ont une origine extérieure.

INDEX

Mots-clés : Linguistique, Néolithique, Néolithisation, Préhistoire 